\title{
Using Facebook Ads to Promote Nutrition and Physical Activity Guidelines Specific to Reproductive and Menopausal Aged Women
}

\author{
Leigh Ann Richardson, MPH, Jessica LC Sapp, DrPH* and Jennifer L Sedillo, PhD \\ Public Health Program, American Public University System, USA \\ *Corresponding author: Jessica LC Sapp, Associate Professor, Public Health Program, American Public \\ University System, School of Health Sciences, Charles Town, WV, USA
}

\begin{abstract}
Background: The aim of this study was to evaluate the use of Facebook ads for reaching women of reproductive and menopausal ages to promote specific nutrition and physical activity recommendations for pregnancy or menopause. This descriptive research study used Facebook ads and posts to deliver an online health campaign.

Methods: The Healthy Adaptations for Life campaign was administered from October 2019 to November 2019. Four Facebook ads were delivered to three target audiences: 1) Women, 18 to 39 years old, located in the U.S. $(n=1), 2)$ women, 18 to 39 years old, located in the U.S. with special interests related to pregnancy $(n=1)$, and 3$)$ women, 40 to 64 years old, located in the U.S. $(n=2)$.

Results: The ads reached 57199 women with 1865 clicks, and $\$ 0.30$ cost per result (CPR). Pregnancy ads had 816 clicks, reached 34 200, and \$0.39 CPR. Menopause ads had 1049 clicks, reached 23 782, and \$0.23 CPR.

Conclusion: Facebook ads can be cost-effective for nutritional guidance during female-specific life events. However, implementation of new knowledge could not be determined.
\end{abstract}

\section{Keywords}

Menopause, Pregnancy, Nutrition, Physical activity, Social media

\section{Introduction}

Nutrition and physical activity are the two major components to living longer and healthier lives [1]. For this reason, many mass media health campaigns incorporate eating healthy and staying physically active into their promotion message. However, these campaigns apply to the population in general and are not tailored to specific groups including pregnant and menopausal women [2-3]. Unlike men, women experience different life events marked by physiological and hormonal changes that require adjustments to their diets and physical activity regimes [4-5]. As women reach reproductive and menopausal ages, poor diet and lack of physical activity can lead to excess weight gain and result in severe and chronic complications that reduce the quantity and quality of women's lives [6-7].

Despite the increased risks associated with excess weight for pregnant or menopausal women, few health promotion campaigns have focused on increasing knowledge about dietary and activity guidelines for these groups. To address this health information gap, the social networking site Facebook was used to deliver healthy diet and physical activity recommendations specific to pregnant or menopausal women via targeted advertisements. The purpose of this study was to evaluate the use of Facebook ads for reaching women of reproductive and menopausal ages to promote nutrition and physical activity.

\section{Methods}

\section{Study overview}

The Healthy Adaptations for Life (HAL) campaign was used to promote nutrition and physical activity among women of reproductive and menopausal age groups. Facebook ads were used to provide information pertaining to weight management techniques via government websites. Two ad sets were created to target each group with a total of 4 ads used in the campaign. For easy recognition and tracking, the hashtag \#Healthy 
Adaptations 4 Life was used on all Facebook ads. The American Public University System (APUS) Public Health Facebook Page was used to disseminate ads and posts for this study. Data were collected through Facebook metrics and were not directly collected from human subjects.

The HAL campaign was part of a larger research study that consisted of 11 social marketing health campaigns. The aim of the original study was to evaluate the effectiveness of digital volunteering for public health community service.

\section{Foundational Social and Behavioral Theories for the Study}

The advertisements for HAL were developed using self-referencing and social cognitive theories. HAL used images related to the target populations to increase interest and draw similar groups to the advertisement. The self-reference effect is when individuals can relate the information being presented (in this case, digital advertisements) to themselves [8]. When individuals relate more strongly to the information, this can lead to more favorable evaluations of the information [8]. Addi- tionally, the social cognitive theory uses peer influence to motivate behavior change [9]. By including various ethnicities and ages in the ads, viewers could either relate to the image based on their own self-reference or the influence of peer groups who mirror positive lifestyle habits [8-9].

\section{Facebook Ads}

Ads were created using stock images, a short message (approximately 280 characters), and a link to a reliable, open sourced website that contained information about health practices. The first ad set targeted women of reproductive ages 18-39 within the U.S. For this ad set, the two ads contained the same messaging, stock image, and link provided (Figure 1). The difference between the two ads was the use of targeting the ad to pregnancy-related interest groups (P-IG) for one ad versus the other ad targeted a generalized group (PG). The pregnancy-related interest groups selected were members of at least one of the following on Facebook: Belly Belly - Pregnancy, Birth \& Parenting, Pregnancy and Newborn Magazine, Pregnancy \& Birth (magazine), Fit Pregnancy, Pregnancy Magazine, Nutrition and pregnancy, Pregnancy, Pregnancy Exercise or Pregnancy

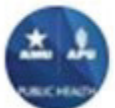

AMU \& APU Public Health

Sponsored 6

\section{Being overweight while pregnant can lead to serious health complications. \#HealthyAdaptations4Life}

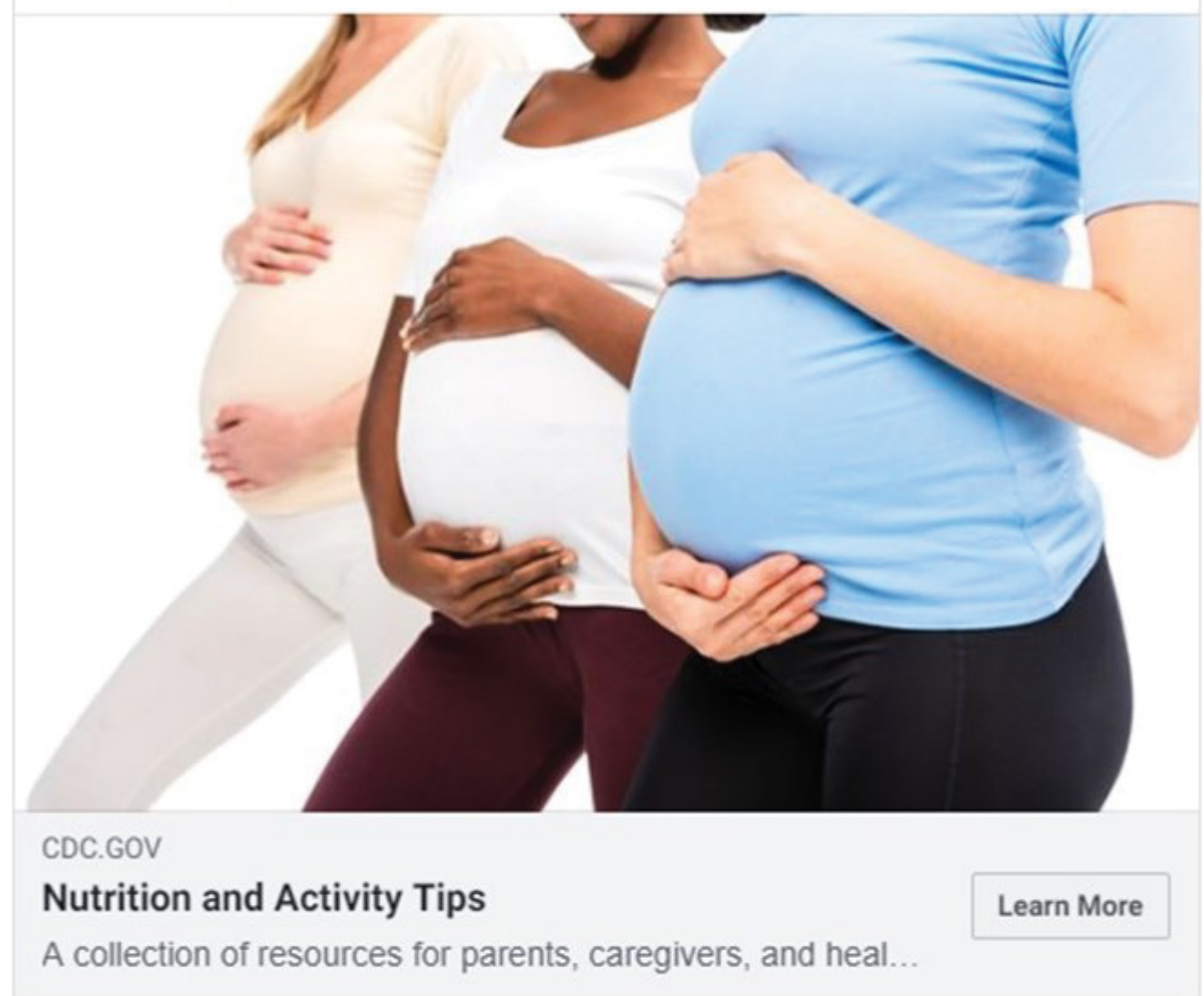

Figure 1: Pregnancy-related facebook advertisement for the health adaptations for life campaign. 


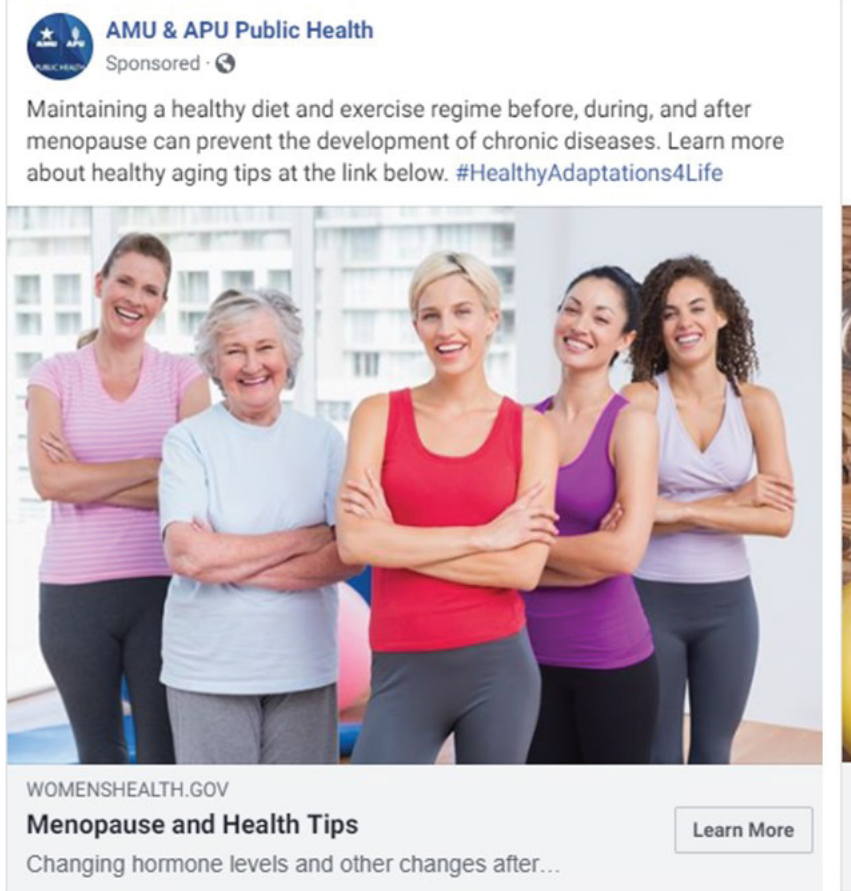

\section{AMU \& APU Public Health Sponsored 6}

Weight gain is common following menopause, but too much weight gain can cause significant health concerns. Learn more tips and information about healthy aging at the link below. \#HealthyAdaptations4Life

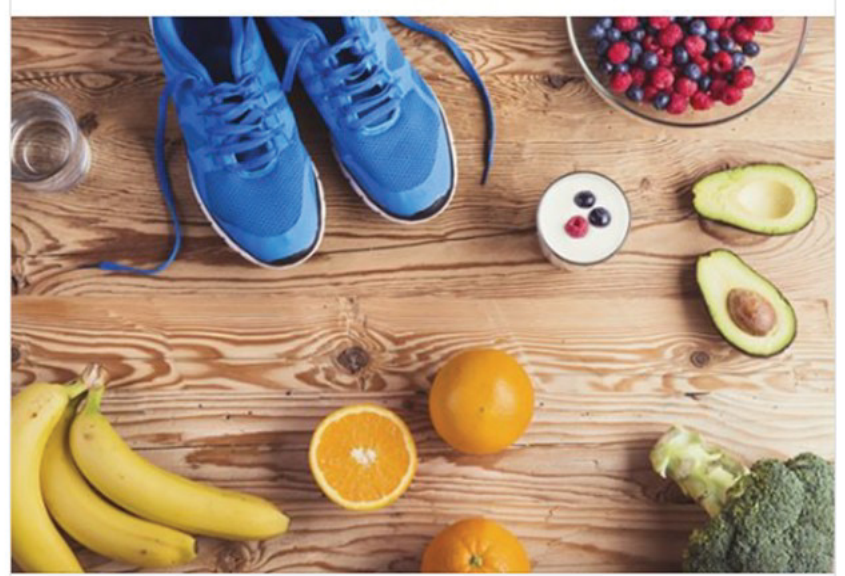

WOMENSHEALTH.GOV

Menopause and Health Tips

Learn More

Changing hormone levels and other changes after.

Figure 2: Menopause-related facebook advertisements (M1 and M2) for the healthy adaptations for life campaign.

Babies and Children's Expo. Collectively, these interest groups had the potential to reach $27,000,000$ people versus $61,000,000$ people for the PG ad parameters. All ad messages were created to fit into Facebook advertisement guidelines. The link was selected from the Centers for Disease Control and Prevention (https:// www.cdc.gov/maternal-infant-toddler-resources).Both ads were delivered from October 3, 2019, to October 10, 2019, using Lowest Bid Strategy with a daily budget of $\$ 20.00$.

The second set of ads targeted women of menopausal age range from 40-64 and located in the United States. Facebook does have interest groups specifically for menopause support; however, the groups are relatively small and did not have a large enough representation to select as an interest group in the development of the ad parameters. Therefore, two ads with differing stock images and messaging were used for the menopause campaign to assess which ad would be more appealing to this target group. The link and information provided were the same: the Office on Women's Health link for menopause information (www.womenshealth. gov/menopause-and-your-health). The Menopause-1 ad(M1) promoted a healthy diet and exercise routine to prevent the onset of chronic diseases and included a stock image of women of various ages and ethnicities in exercise attire who could represent menopausal aged women (Figure 2). The Menopause-2ad(M2) related to weight gain during menopause and included a stock image of healthy foods and tennis shoes as indicators of a healthy lifestyle (Figure 2). The ads ran from October 28,2019 , to November 6,2019, with a daily budget of $\$ 25.00$.

\section{Measures}

To assess the effectiveness of the HAL campaign, a comparative analysis of objective measures collected by Facebook Ads Manager was used. The following definitions were developed by Facebook for the measures collected:

- Reach: The number of people who saw an ad at least once.

- Cost per Result (CPR): The average cost (USD) per result (link clicks) from an ad.

- Frequency: The average number of times each person saw an ad.

- Link Clicks: The number of clicks on links within the ad that led to destinations or experiences, on or off Facebook.

\section{Results}

The HAL campaign resulted in 1865 link clicks, reached 57199women, averaged a CPR of $\$ 0.30$, and cost $\$ 561.65$ (Table 1). No comments were made on the ads and the only reactions $(n=50)$ were the like and love options (Facebook offers six reactions: like, love, haha, wow, sad, angry).

The pregnancy ad set resulted in 816 link clicks, an average CPR of $\$ 0.39$, reached 34200 women, and cost $\$ 315.29$. P-IG had a CPR of $\$ 0.37$ versus the PG CPR of $\$ 0.41$, which when compared with the number of link clicks resulted in a similar ad cost of $\$ 158.81$ and $\$ 156.48$, respectively. The spread in age groups was similar among both ads with both groups having the most reach and link clicks from the 25-34-year-old group, but 
known, it could aid future pregnancy-related health campaigns to focus more on image selection, message delivery, and content shared versus the use of interest groups.

Additionally, the data collected from the pregnancy ad set gave more insight into societal changes regarding women and pregnancy. Research has shown that women are waiting until later in life to have their first child [15-16]. In 2014, 42.1\% of first-time mothers were 24 years old or younger, $48.8 \%$ of first-time mothers were 25-34 years old, and $9.1 \%$ of first-time mothers were 35 and older [16]. From the pregnancy ad set, as age increased, both reach and link clicks also increased (resulting in lower (PR) which coincides with previously published data showing that age groups 25-34 and 3544 use Facebook at greater proportions than 18-24 year olds [17]. However, in both groups, women aged 35-44 had the lowest CPR (higher interest) even when compared to the 25-34 year old group (P-IG \$0.34 v. \$0.37 and PG \$0.38 v. \$0.42).

The menopause ad set provided two different ads to the same targeted audience and received similar CPR, but varied drastically on the number of women reached. Facebook mentions that the bid, budget, and audience targeting may influence the reach of an ad; however, these factors were controlled throughout the campaign and within ad sets. Both ads were released on the same day with the same duration. The variation in reach could be due to the ads' performance in the first few days. M1 received 14,25 , and 21 link clicks within the first three days and $M 2$ received 67,76 , and 88 link clicks in the first three days. Therefore, the performance of the ads within the first three days of being published may have influenced the number of people reached. The ad with more link clicks (M2) in the beginning was shared more widely with the target audience.

The initial hypothesis during the creation of the menopause ad set was women would be more drawn to M1. By showing women of various ages and ethnicities, it was hypothesized more women would relate or see a representation of themselves in the ad (self-reference theory). In contrast, M2's image showed only items associated with a healthy lifestyle: Tennis shoes and healthy fruits and vegetables. Messaging was also different with $\mathrm{M} 1$ focusing on prevention techniques (diet and exercise) and $\mathrm{M} 2$ highlighting an outcome (weight gain) of poor lifestyle habits. M2s ad success may be attributed to the inclusion of two primary factors: visual representation of positive lifestyle behaviors, which can be associated with the preparation stage of the trans theoretical model, and the phrasing of a common health topic among menopausal aged women (weight gain), which may relate to the target audience (self-reference theory).Despite the differences applied to these ads, no major differences were seen across the targeted age groups based on CPR (with the exception of age group
35-44 for the M1 ad which only had 1 click).The lowest CPR for both ads aligned with the average age of onset for menopause (45-55 years old) [18]. This substantiates previous research that menopausal aged women are interested in gaining information about what they can change or modify to improve their health [19].

The purpose of this study was to evaluate the use of Facebook ads for reaching women of reproductive and menopausal age to promote weight management. The use of Facebook ads as the medium for the educational material allowed for tailored messaging and data collection on each ad. Approximately $75 \%$ of pregnant women use the Internet to search for pregnancy-related topics with fetal development and nutrition information being the most searched topics [12]. Menopausal aged women "account for over half (53\%) of core female social media users in the U.S." and are regularly searching for health information [19]. Overall, the HAL campaign proved that reproductive and menopausal aged women are interested in gaining information about weight management for these two female specific life events and are accepting of social media as a reliable source. With information on the Internet being unregulated, misleading, and often unreliable, using Facebook ads to serve as a reliable source for accurate health information pertaining to healthy weight management was shown to be valued by the target audiences.

\section{Limitations}

There are limitations to this research that are intrinsic to using social media. Aside from the parameters established in the beginning stages of ad development, no additional information was collected on the target populations. Facebook does not make available in Facebook Ads Manager demographic information on race and ethnicity, so it is unknown if the advertisements reached certain racial or ethnic groups more than others. Age groups afford some insight, but it does not provide a comprehensive picture of the target audience or those that performed a link click. Facebook Ads Manager uses defined age groups for data reporting. Although ads were tailored to mid-range ages of 35-44, (ie, 39 and 40), data are reported in the 35-44 age bracket. It is expected that the maximum age of Facebook users reached for pregnancy ads was 39 and minimum age of menopause ads was 40 based on the target audiences, but the data were reported as $35-44$ years old. The use of Facebook advertisements limits the audience to those with computer or internet access and Facebook accounts. In addition, the campaign duration limited the users reached to those that accessed social media accounts during this time period.

Facebook does not allow for language or ads that it considers too invasive to users (eg, Are you pregnant?), even though this may be common usage in health education materials. Although Facebook advertisement 
regulations prevent companies from targeting certain demographics unfairly, it can be an issue for public health officials who are trying to target specific demographics due to their elevated risks of a disease and to share health information specific to the target population.

\section{Implications for Research and Practice}

Health promotion is a major function of public health and encourages positive health behaviors to include nutrition and exercise practices to prevent the development of avoidable diseases. From the results of the HAL campaign, future pregnancy-related promotion should focus on the ad development process rather than targeting specific pregnancy-interest groups. The data show lower interest in pregnancy-related weight management information from younger women. More studies are needed to identify if this is a common theme among younger women and how to reach the younger at risk population. More research is needed to identify the best modes of communicating these topics and adoption of healthy behavior changes for this at risk group of women.

In this study, older women (ages 45-64) showed the highest interest in health knowledge seeking. While pregnancy health and diet are prominent topics in health campaigns, diet and health during menopause is often overlooked. Through the use of social media, information on this important female specific life event can be promoted and targeted to the population most at risk.

This study demonstrates that Facebook ads can be a cost-effective approach to disseminating health education and promotion to a wide audience. Facebook has become a more widely used outlet to create and implement public health messaging. The features that make it a great tool include the ability to precisely target the desired audience, the relatively low costs, and the metrics and demographic information that it provides.

\section{Conflicts of Interest}

None Declared.

\section{References}

1. U.S. Department of Health and Human Services. Nutrition, Physical Activity, and Obesity.
2. Wong FL, Greenwell M, Gates S, Berkowitz JM (2008) It's What You Do! American Journal of Preventive Medicine. 34: 175-182.

3. (2020) US Department of Health and Human Services. Walk. Run. Dance. Play. What's your move? Move Your Way.

4. Kominiarek MA, Rajan P (2016) Nutrition Recommendations in Pregnancy and Lactation. Med Clin North Am 100: 1199-1215.

5. (2020) US Department of Health and Human Services. Weight loss and women.

6. Stubert J, Reister F, Hartmann S, Janni W (2018) The Risks Associated With Obesity in Pregnancy. Dtsch Arztebl Int 115: 276-283.

7. Teede HJ, Lombard C, Deeks AA (2010) Obesity, metabolic complications and the menopause: An opportunity for prevention. Climacteric 13: 203-209.

8. Lee CK-C, Fernandez N, Martin BA (2001) Using self-referencing to explain the effectiveness of ethnic minority models in advertising. International Journal of Advertising 21: 367-379.

9. Bandura A (1977) Social Learning Theory. Englewood Cliffs, NJ: Prentice Hall.

10. Graham JE, Moore JL, Bell RC, Miller T (2019) Digital marketing to promote healthy weight gain among pregnant women in alberta: An implementation study. Journal of Medical Internet Research 21.

11. Irvine M (2018) Facebook Ad Benchmarks for YOUR Industry [Data]. Word Stream.

12. Sayakhot $P$, Carolan-Olah M (2016) Internet use by pregnant women seeking pregnancy-related information: A systematic review. BMC Pregnancy and Childbirth 16.

13. ht t p s://support.google.com/adsense/answer/7549925?hl=en.

14. https://www.facebook.com/policy/cookies/.

15. Livingston $\mathrm{G}$ (2018) US women are postponing motherhood, but not as much as those in most other developed nations. Fact Tank News in the Numbers.

16. Mathews T, Hamilton B (2016) Mean Age of Mothers is on the Rise: United States, 2000-2014. NCHS Data Brief.

17. https://www.statista.com/statistics/187041/us-user-age-distribution-on-facebook/.

18. (2017) US Department of Health and Human Services. What Is Menopause? National Institute on Aging.

19. Washington O (2010) The Center for Social Impact Communication at Georgetown University. Using Social Media Platform to Amplify Public Health Messages. 